\title{
Comparison of the Passive Dynamics of Walking on Ground, Tied-belt and Split-belt Treadmills, and via the Gait Enhancing Mobile Shoe (GEMS)
}

\author{
Ismet Handžić and Kyle B. Reed \\ Department of Mechanical Engineering \\ University of South Florida \\ Tampa, FL, 33620, U.S.A.
}

\begin{abstract}
This research compares walking over ground, on a split-belt treadmill, on a tied-belt treadmill, and on the Gait Enhancing Mobile Shoe (GEMS) in both humans and simulated on a passive dynamic model. Passive Dynamic Walkers (PDW) have been researched for decades, yet only recently has the model been used significantly in gait rehabilitation. We aim to identify how well the two-dimensional PDW can be used as a kinematic approximation tool for gait analysis. In this work, the PDW was scaled according to an anthropomorphic human model. For comparison, measurements were taken of humans walking in the same four environments. For normal walking, the PDW was found to be a good approximation for symmetric and rhythmic hip position, foot position, and velocity profiles. Tiedbelt and split-belt treadmill model estimations revealed that the PDW's lack of dorsiflexion, joint stiffness, and joint damping limited the comparison, however trends between the human and the model agreed. The kinematics of the GEMS showed good agreement in interlimb interactions indicating that the PDW can be used as a good kinematic predictor for the GEMS.
\end{abstract}

\section{INTRODUCTION}

This research focuses on the passive dynamics of gait separate from the cognitive influences associated with walking. We aim to determine how the passive dynamics affect the gait so rehabilitation methods can be evaluated prior to testing on humans. In this paper, we compare the passive dynamics to humans walking in four environments: (1) over ground, (2) on a tied-belt treadmill, (3) on a splitbelt treadmill [1][2][3][4][5], and (4) on the Gait Enhancing Mobile Shoe (GEMS) [6][7][8][9].

The passive dynamics are modeled based on a passive dynamic walker (PDW) model [21]. A PDW is a biped that walks down a decline solely by the force of gravity [10]. In contrast to humanoid robots, the PDW shows a repeatable human-like gait, walking with a near energy optimal gait. An important characteristic of PDWs is that the focus is on the dynamic effects of a gait, excluding the cognitive aspects. For a rational comparison, the PDW model parameters used for this analysis are extracted from an anthropomorphic model [11] and proportionally sized such that the masses and mass distribution are relatively sized down from an individual (Figure 1). While this walking model has limitations, it does give further insight into gait and gait rehabilitation.

Hemiplegia, the paralysis of one side of the body, is often subject to gait rehabilitation and is often caused from stroke and/or central nervous system damage and can result in an asymmetric gait [1][2][3][4][5]. These gait asymmetries can be modeled using a PDW model by varying mass and mass distribution parameters [12]. Current methods used to change

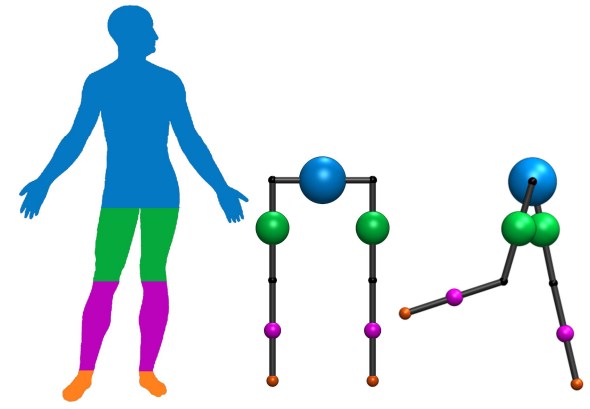

Fig. 1. PDW model matching the anthropomorphic model was used to

interlimb coordination include using conventional treadmills, split-belt treadmills, and the GEMS.

First, we focus on explaining our PDW computer model structure and parameters. Next, we compare a normal, undisturbed, steady state, and symmetric PDW gait to a recorded normal human gait. We then explain and discuss our results of the PDW model in the four walking conditions.

\section{BACKGROUND}

\section{A. Normal Walking}

One stride is the sum of a left and right step and is divided into two distinct phases: stance phase (foot is in contact with the ground), and swing phase (foot swings by the hip). The swing phase is initiated by the toe-off (pre-swing) and is terminated by the heel strike (initial contact). As the foot approaches heel strike, the knee locks (knee strike) to a stiff straight pose with a mean knee posture of $5^{\circ}$ flexion, however subsequent to toe-off knee flexion can reach up to $60^{\circ}$ [15]. Normal human gait cycle includes a double support phase during which both feet are simultaneously on the ground. Double support accounts for $\sim 10 \%$ of the gait cycle [15].

Tied-belt treadmills are conventional treadmills with one belt which equally pushes both feet backward during stance phase and are often found in athletic gyms. Tied-belt treadmill walking is commonly equated to normal walking, given that the kinematics and kinetics of overground and treadmill locomotion are quite comparable [24][25].

\section{B. Gait Rehabilitation}

Individuals suffering gait impairment from central nervous system damage often require gait training on a tiedbelt and/or split-belt treadmill. A split-belt treadmill has two independently moving belts for each foot, allowing the lagging foot to be pushed back at a faster speed than the opposite foot. Walking on a split-belt 
treadmill changes the interlimb coordination in individuals with hemiparesis [1][2][3][4][5]. Pathological gait such as hemiplegia is often caused by stroke and can result in spatial and temporal gait asymmetry [14]. An asymmetric gait occurs when the step length of the left foot does not equal the step length of the right foot, resulting in a limping walking pattern. This exaggerated motion, such as the motion created by split-belt treadmills, creates a desired symmetric after-effect in gait [1]. Gait Rehabilitation can greatly benefit from a human gait model that can approximate normal gait, pathological gait such as hemiplegia, or modifications and perturbations to human gait. Although a split-belt treadmill walking study using a biped humanoid quasi-static robot has been demonstrated [10], a PDW model can present a more realistic view of the human's gait dynamics. This advantage make PDWs more attractive to human gait and gait rehabilitation analysis.

Altering the coordination between two legs (interlimb coordination) in adults can also be achieved through a novel device, the Gait Enhancing Mobile Shoe (GEMS) (Figure 2), that kinetically and kinematically acts similar to a split-belt treadmill [6][8][9]. The GEMS aims to make the rehabilitative motions of a split-belt treadmill available over longer periods of time and in various environments, exploring long-term effects. Studies on healthy individuals have indicated that wearing the GEMS alters gait in a similar fashion as a split-belt treadmill [6][8].

\section{Passive Dynamic Walking}

A PDW is an entirely mechanical device that is able to exhibit a steady and stable gait down a decline purely due to gravitational forces and no other energy input. The energy gained by its progression is lost during two inelastic collision events: knee strike and heel strike. Post collision velocities are calculated using conservation of angular momentum. The walker continuously completes two stages in its dynamics: a three-link phase (pre-knee lock swing phase) and a twolink phase (post-knee lock swing phase). The dynamics of the PDW model can be described with the Lagrangian formulation of a multi-pendulum system shown in [19][23]. The two-dimensional PDW concept was initially analyzed as a rimless wheel progressing down a slope [17], then was developed into a double pendulum model [18], and knees were added [19]. By distinguishing left and right legs and varying leg mass and mass distribution, Honeycutt et al. [21]

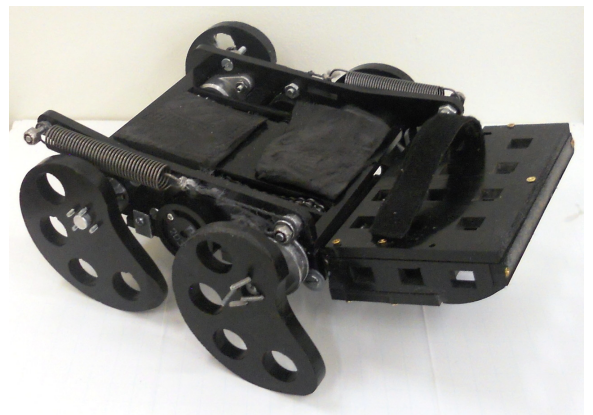

Fig. 2. The Gait Enhancing Mobile Shoe (GEMS) mimics the rehabilitative motions of a split-belt treadmill. furthered the two-dimensional kneed PDW, allowing for an asymmetric gait analysis of the PDW. Recently the PDW has been put to use in practical applications such as an actuated PDW to compensate for gravity [20], a steering PDW [21], and prosthetics [22].

\section{PDW MODEL}

While many gait rehabilitation studies have been conducted on individuals, it is advantageous to use a model that can separate the cognitive effects from the dynamics. Human walking control represents the modification of energy input and disbursement through muscle action, joint damping, and joint stiffness. However, we are looking at steady state dynamics that occur during steady state walking with permanent perturbations. In this way, the dynamics can be altered that will necessitate the desired cognitive changes and after-effects when applied to humans. To model the passive dynamics and evaluate how to apply the needed perturbations, we will use the asymmetric PDW Model outlined by Honeycut et al. [12] to analyze and compare the behavior of several controlled perturbations to the same perturbations on a human individual.

The model captures certain aspects of gait despite several limitations. The benefit of this model is that we can change the model parameters such as limb length, masses, and mass distribution, all of which affect the PDW gait stability and symmetry, yet are difficult to change in humans. However, the PDW model does not account for dorsiflexion, joint stiffness, and joint damping nor does it exhibit a double support phase during walking. Since the PDW models a human body from the hip and below, only the masses and mass distribution of the upper body, thighs, shanks, and feet are considered. In this paper we define upper body to include the head, neck, and trunk without the arm limbs. Despite the limitations, being able to quickly and easily change these parameters makes the model useful for asymmetric gait analysis and gait rehabilitation research [22].

\section{A. Asymmetric Seven Mass Model}

Honeycut et al. [12] describe a nine mass PDW model, however, due to the nature of our research we only employed seven of the nine masses, setting two of the four thigh masses to zero and moving the lower shank mass down to represent the foot mass. As shown in Figure 1, the seven mass model relates directly to the trunk, thigh, shank, and foot masses. This mass distribution is analogous to anthropomorphic data [11]. Consequently, this mass distribution enables us to add weight to the foot mass, representing a GEMS.

\section{B. Model Scaling}

For the purposes of this research, it is important to match and properly scale the PDW model to an individual. This allows for a rational comparison and judgment on the validity of the model. For correct scaling we use the anthropomorphic data which outlines averaged masses and mass distributions for a human upper body, thigh, shank, and foot [11]. According to Perry et. al. [15], the upper body 
TABLE I

PARAMETERS OF THE HUMANS AND MODELS USED FOR THIS COMPARISON.

\begin{tabular}{|c|l|l|l|l|l|l|}
\hline & \multicolumn{2}{|l|}{$\begin{array}{l}\text { Anthropomorphic Model Mass } \\
\text { for Person (kg) }\end{array}$} & \multicolumn{2}{l|}{$\begin{array}{l}\text { Anthropomorphic Model Mass } \\
\text { for PDW model (kg) }\end{array}$} & \multicolumn{2}{l|}{$\begin{array}{l}\text { Anthropomorphic Model Segment } \\
\text { COM Distance From Hip (m) }\end{array}$} \\
\hline & Human & \% Total & Human & \% Total & Human & Model \\
\hline Upper Body & 52.90 & $58.32 \%$ & 4.00 & $57.31 \%$ & 0.00 & 0.00 \\
Thigh & 24.40 & $26.90 \%$ & 1.90 & $27.22 \%$ & 0.22 & 0.22 \\
Knee & 0.00 & $0.00 \%$ & 0.00 & $0.00 \%$ & 0.50 & 0.49 \\
Shank & 9.23 & $10.18 \%$ & 0.60 & $8.60 \%$ & 0.72 & 0.72 \\
Foot & 2.91 & $3.21 \%$ & 0.22 & $3.15 \%$ & 0.98 & 1.00 \\
Full Body & 90.7 & $100.00 \%$ & 6.98 & $100.00 \%$ & 1.85 & 1.00 \\
\hline
\end{tabular}

travels as a unit during normal gait and only moves up and down, hence, the upper body, which includes head, neck, and trunk is represented in our PDW model as only the hip mass.

Our PDW model is one meter in height (ground to hip) while the hip height for the tested individuals is also approximately one meter. In conjunction with the anthropomorphic data, this yielded a scaled down mass distribution between the tested individuals and PDW model, scaling the thigh, shank, and foot mass accordingly. Table I shows the masses and mass distribution of human measurements for normal walking and corresponding PDW model values. Due to instability in walking with perturbations, the shank mass on the PDW model was decreased $15 \%$ from $0.70 \mathrm{~kg}$ to $0.60 \mathrm{~kg}$.

\section{PDW Stability Criteria}

Steady state measurements were taken of a stable walking model for each of the four walking environments. The stability criteria was defined as fifty steps without the PDW tumbling or with foot drag. To ensure steady state, the model results were taken during stride number thirty-five and thirty-eight. During each trial the sagittal plane position and velocity of the heel and hip mass were recorded. The PDW ramp angle used for evaluation was $3.2^{\circ}$, unless stated otherwise, which was generally most stable.

\section{EXPERIMENTAL CONDITIONS}

In addition to normal walking, our seven mass model enables testing on three additional setups: tied-belt treadmill, split-belt treadmill, and onto a motion that resembles a GEMS. For clear interpretation and comparison of normal and PDW model gait behavior, we show the position and velocity versus time data for each environment for each heel.

All participants read and signed a University of South Florida IRB approved consent form prior to participating.

\section{A. Normal Walking}

1) Human: Measurements for humans walking normally were taken by a 3D VICON motion capture system infrared camera at $120 \mathrm{~Hz}$ at the University of South Florida. Measurements were taken in the sagittal plane, capturing strides three and four out of five total. The recorded individual had markers placed on the left and right ilium of the hip bone and on the lowest point on the back of the subject's sneaker of both feet. The subject was age 26 , male, $1.85 \mathrm{~m}$ in height, with a weight of $90.7 \mathrm{~kg}$.
2) Model: Normal walking model used the anthropomorphically scaled PDW model is described in Section III.

\section{B. Tied-belt}

1) Human: Human measurements for tied-belt walking were taken at Moss Rehabilitation Research Institute using CODAmotion infrared motion system at $100 \mathrm{~Hz}$ in the sagittal plane. Tied-belt and split-belt data were both recorded on a $1.49 \mathrm{~kW}(2 \mathrm{hp})$ WoodWay split-belt treadmill. The subject for tied-belt and split-belt treadmill was age 30 , male, $1.81 \mathrm{~m}$ in height, with a weight of $81.6 \mathrm{~kg}$. The tiedbelt treadmill was run at a constant $0.48 \pm 0.02 \mathrm{~m} / \mathrm{s}$. To match walking data, a speed equivalent to the recorded negative belt speed was added; thus, their walking speed is comparable to walking over ground at the same speed as the treadmill.

2) Model: The PDW model was modified so that the ramp it is walking on constantly moves at a set velocity. Each time a leg is in stance phase, a steady backward velocity is applied, displacing the foot backwards like a treadmill. One of the feet is always in contact with the ground and that foot experiences a constant backward velocity. The velocity magnitude for the tied belt treadmill was the mean of the treadmill speed used in weight-supported treadmill training for rehabilitation [16] scaled down to match the model.

\section{Split-belt}

1) Human: Split-belt walking measurements were taken in the same location, setup, and fashion that the tied-belt measurements were taken. The slower belt on the split-belt treadmill was run at a constant $0.48 \pm 0.02 \mathrm{~m} / \mathrm{s}$, while the faster belt was run at $1.45 \pm 0.03 \mathrm{~m} / \mathrm{s}$. Again, as with the tied-belt treadmill data, we standardized the collected splitbelt data by adding an equivalent amount of belt speed to the recorded negative belt speed.

2) Model: The model is able to simulate a PDW walking on a split-belt treadmill by treating each tread independently. As only one tread has a greater relative velocity than the other, we set one tread to zero and move the opposite tread at a constant velocity during the stance phase. The velocity magnitude for the split-belt treadmill was determined using a split-belt treadmill adaptation study for post-stroke patients [1]. The velocity is the mean walking speed of the hemiplegic affected individuals.

\section{GEMS}

1) Human: This study was performed in the same location and fashion as normal walking, but while wearing the GEMS. 
TABLE II

SUMMERY OF TEMPORAL AND SPATIAL WALKING PARAMETERS IN HUMAN AND PDW MODEL

\begin{tabular}{|l|l|l|l|l|l|l|l|l|l|l|l|l|}
\hline & \multicolumn{3}{|c|}{ Normal Walking } & \multicolumn{3}{c|}{ Tied-belt Walking } & \multicolumn{3}{c|}{ Split-Belt Walking } & \multicolumn{3}{c|}{ GEMS Walking } \\
\hline & Human & PDW & Diff & Human & PDW & Diff & Human & PDW & Diff & Human & GEMS & Diff \\
\hline Left Step Length (m) & 0.73 & 0.59 & $21 \%$ & 0.82 & 0.45 & $58 \%$ & 0.53 & 1.01 & $62 \%$ & 0.76 & 0.46 & $49 \%$ \\
\hline Right Step Length (m) & 0.73 & 0.59 & $21 \%$ & 0.82 & 0.45 & $58 \%$ & 1.02 & $11 \%$ & $11 \%$ & 0.51 & 0.7 & $31 \%$ \\
\hline Gait Cycle (s) & 1.24 & 1.42 & $13 \%$ & 1.58 & 1.10 & $35 \%$ & 1.18 & 1.15 & $3 \%$ & 1.19 & 1.32 & $10 \%$ \\
\hline Left Swing Time (s) & 0.62 & 0.71 & $13 \%$ & 0.78 & 0.55 & $34 \%$ & 0.38 & 0.51 & $29 \%$ & 0.51 & 0.53 & $3 \%$ \\
\hline Right Swing Time (s) & 0.62 & 0.71 & $13 \%$ & 0.8 & 0.54 & $38 \%$ & 0.73 & 0.64 & $13 \%$ & 0.68 & 0.74 & $8 \%$ \\
\hline Walking Velocity (m/s) & 0.98 & 0.82 & $17 \%$ & 0.5 & 0.83 & $49 \%$ & 0.5 & 0.88 & $55 \%$ & 0.63 & 0.83 & $27 \%$ \\
\hline Left Maximum Velocity (m/s) & 3.91 & 2.82 & $32 \%$ & 1.92 & 2.93 & $41 \%$ & 2.23 & 3.01 & $29 \%$ & 3.28 & 2.87 & $13 \%$ \\
\hline Right Maximum Velocity (m/s) & 3.89 & 2.83 & $31 \%$ & 1.84 & 2.93 & $45 \%$ & 2.8 & 2.91 & $3 \%$ & 2.5 & 2.58 & $3 \%$ \\
\hline
\end{tabular}

2) Model: Like a split-belt treadmill, the GEMS pushes one foot backward relative to the opposite foot (faster belt). As per its design, the shoe pushes the user's foot back only a limited distance before smoothly bottoming out and coming to a halt so the user can toe off. We proportionally scaled down the mass of the GEMS, but because the size of the PDW model is closely matched to our test subject, the dimensional aspects such as height remain constant. To model the GEMS, its mass, velocity, and travel distance were scaled to the size of the PDW at $7 \%$. The actual GEMS weight of $1.65 \mathrm{~kg}$ was scaled down to $7 \%$ of actual to $0.12 \mathrm{~kg}$ and added to the right foot mass of the PDW. GEMS velocity was scaled from $0.35 \mathrm{~m} / \mathrm{s}$ to $0.025 \mathrm{~m} / \mathrm{s}$ and the GEMS fixed travel distance from $0.15 \mathrm{~m}$ to $0.01 \mathrm{~m}$.

\section{RESULTS AND COMPARISONS}

These results compare the differences between the PDW model (passive) and a human walking (active), thus we do not expect results to align perfectly. We aim to understand the effect that the passive dynamics have on the gait pattern separate from the cognitive influences. This understanding can help us to validate the use of a PDW model for gait pattern prediction. The temporal and spatial values for human and PDW model are summarized in Table II.

\section{A. Normal Walking and Tied-belt Walking}

Normal human walking kinematics has a rhythmic, repetitive, and symmetric motion. As observed in Figure 3, both human and model results follow this description and are very comparable, however velocity of the model is $83 \%$ of the human. The PDW model is completely passive and does not rely on any energy input such as human walking controls, hence the slower PDW walking velocity down the ramp. Normal walking in the model and human were both symmetric in swing time and step length. Both temporal and spatial aspects of walking in PDW and human walking were within $13 \%$ and $21 \%$, respectively.

There is a variation in the model's velocity profile during three-link phase. As the foot approaches knee strike, the human data has a slight decrease in velocity while the model velocity at the same instance in the gait cycle has a minor increase with a steady increase until knee strike. This difference can be explained by the kinematics of the opposite leg and the nature of the PDW model. At that instant the human data velocity decrease is caused by stance leg dorsiflexion of the ankle when the tibialis anterior (frontal shank) muscles contract, while the posterior shank
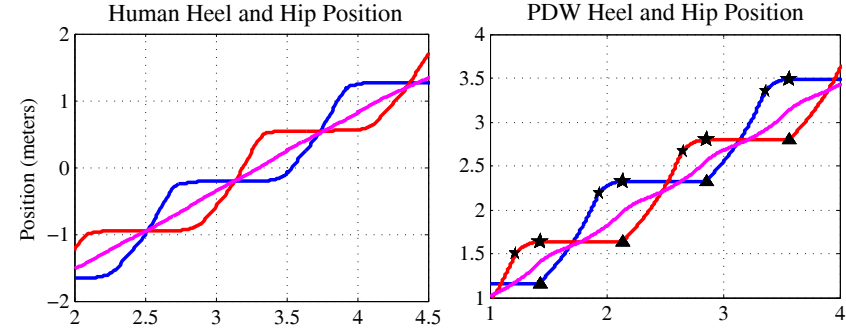

Human Heel and Hip Velocity

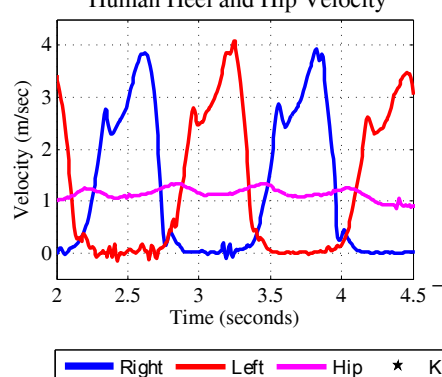

(a)
PDW Heel and Hip Velocity

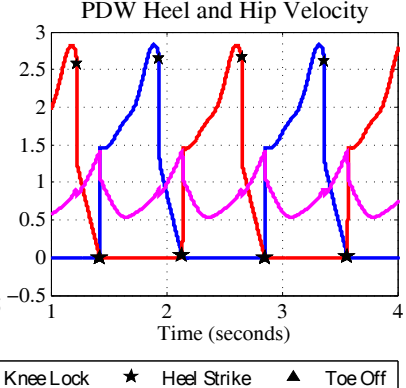

(b)
Fig. 3. Normal human walking data as captured by a motion system (a) compared to steady state PDW walking down a decline (b)

muscle group stretches, dissipating and storing kinetic energy and creating potential muscle energy for toe-off (energy conservation) [15]. This generates an elastic recoil, preparing the limb for swing phase. As our PDW model does not have ankles, this "push-off" phenomenon does not occur.

While wavering of the hip's velocity profile happens during the same instances in the gait cycle as the model, the model's hip velocity profile is more exaggerated, with velocity fluctuation of $40 \%$ for the model and $10 \%$ for a human. This higher fluctuation is explained by the PDW movement down a ramp and dorsiflexion. Unlike horizontal walking, the PDW hip drops the extra distance down the ramp subsequent to knee strike and prior to heel strike. Also, during the gait cycle in human walking, the ankle flexes just before toe off and recoils during toe off. This movement creates a smoother transition between stance and swing phase.

It is interesting to note that hip velocity fluctuation in human walking on the tied-belt treadmill is more pronounced than when walking over ground; walking on the treadmill more closely resembles the hip velocity of the PDW in both normal and tied-belt walking.

In order to further investigate the similarities between human walking and PDW walking, we compared walking on an incline. The ramp angle of the PDW was decreased from 

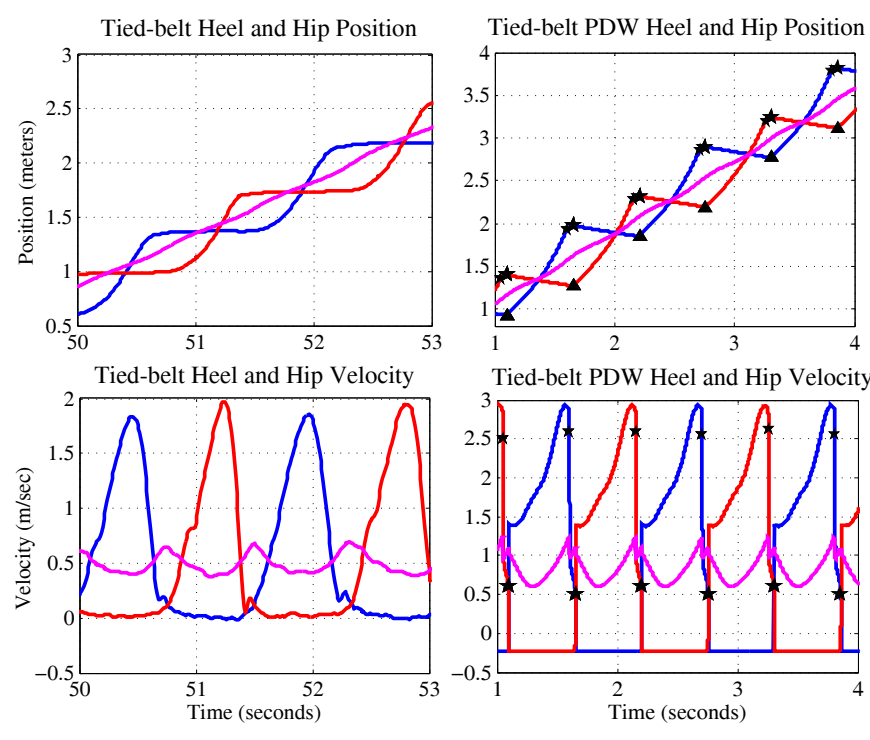

$\longrightarrow$ Right $\longrightarrow$ Left $=$ Hip $\quad$ knee Lock

(a)

(b)

Fig. 4. Kinematics of tied-belt walking for (a) human and (b) PDW model.

$3.0^{\circ}$ to $3.4^{\circ}$ at $0.1^{\circ}$ intervals and compared to A.S.McIntosh et. al.'s [26] human trial data which measured duration, stride length, and walking speed of walking on a $0^{\circ}$ to $10^{\circ}$ decline. The PDW model gait cycle duration, stride length, and walking speed trends matched that of human data. As walking surface slope decreased, both human and model stride length and walking speed increased, while both human and model gait cycle time decreased.

Due to PDW walker instability, tied-belt velocity could not be fully simulated at a properly scaled model velocity of $0.48 \mathrm{~m} / \mathrm{s}$. Instead walker tied-belt velocity was iterated to find a maximum possible speed of $0.23 \mathrm{~m} / \mathrm{s}$ ( $52 \%$ less). For consistency and comparison, human tied-belt data was normalized to a non-zero hip velocity. Figure 4 shows both human and modeled tied-belt walking.

While human and model walking parameters are comparable, spatial and temporal walking characteristics between tied-belt human and model walking show a greater difference with gait cycle time, step length, and maximum foot velocity difference of $35 \%, 58 \%$, and $41 \%$, respectively. Although this difference seems significant, one must consider that the belt speed was run at nearly half the speed due to walker instability.

\section{B. Split-belt Walking and GEMS Walking}

As shown in Figure 5, the PDW model could not simulate a scaled down split-belt treadmill velocity of $0.78 \mathrm{~m} / \mathrm{s}$ due to instability. Using appropriate anthropomorphic data, the PDW tied-belt and split-belt speed was iterated to find a maximum split-belt speed of $0.25 \mathrm{~m} / \mathrm{s}$ ( $75 \%$ less). However, asymmetric trends persisted in the temporal domain. With respect to temporal parameters, the model's asymmetry followed the same trend with a right swing phase and left swing phase.

When wearing the GEMS, position and velocity profiles for the human and the model data yielded asymmetric

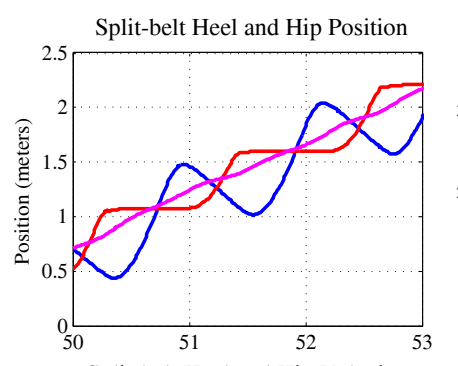

Split-belt PDW Heel and Hip Position

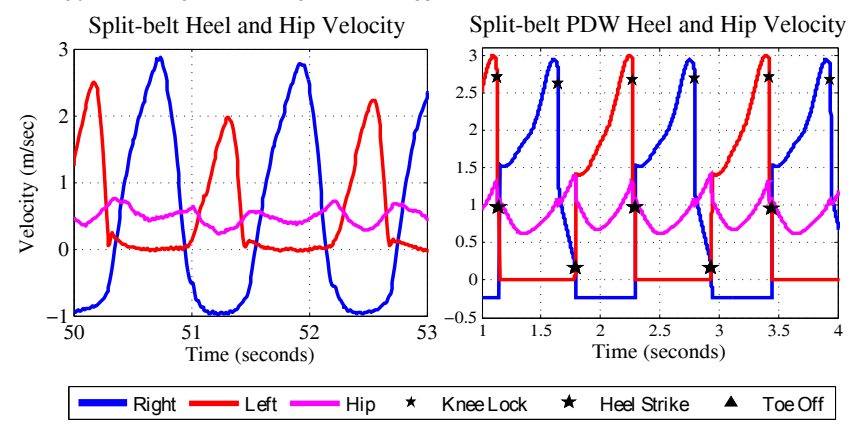

(a)

(b)

Fig. 5. Kinematics of split-belt walking for (a) human and (b) PDW model.
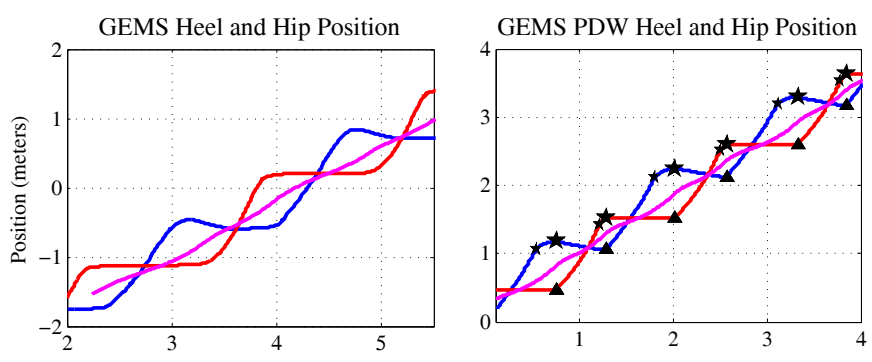

GEMS Heel and Hip Velocity

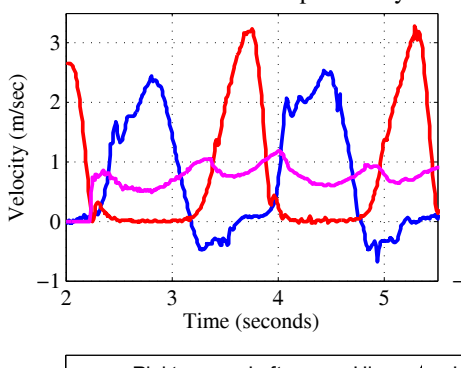

GEMS PDW Heel and Hip Velocity

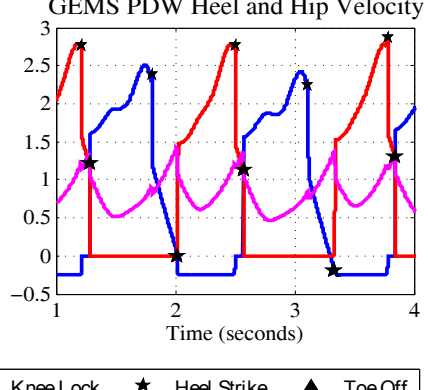

(a)

(b)

Fig. 6. Measured (a) and PDW modeled (b) walking kinematics with the Gait Enhancing Mobile Shoe (GEMS)

tendencies as expected. As seen in Figure 6, human and modeled profiles are very comparable in shape. As the GEMS pushes the right foot backward, the left foot compensates by attaining a $25 \%$ greater peak velocity during swing phase. This difference in velocities is only $13 \%$ in the simulated data. Also during the right foot swing phase with the GEMS, both human and simulated velocity profiles take a slight dip prior to knee strike and do not summit as sharply as the opposite foot velocity without the GEMS. This dip is largely due to the extra weight of the shoe.

While the test subject substantially compensated for the backward GEMS movement by a faster forward step of the opposite leg and in turn a faster hip progression, the model's 
hip does not portray this. The simulated model hip movement shows the same trend subsequent to GEMS movement, and it kept a constant maxima and minima.

Swing duration trends agree between human and model. Both show a greater swing time on the GEMS foot in comparison to the opposite foot, $25 \%$ and $28 \%$ increase respectively. However, the step length difference trend between human and model varied. Although the individual's right step length was $32 \%$ lower than the left, PDW model right step length was $29 \%$ higher than the left.

\section{CONCLUSion AND Future WORK}

We presented the PDW walker as a rehabilitation oriented research tool, comparing kinematic characteristics of normal gait, split-belt and tied-belt treadmill walking, and walking with the GEMS. Although the model position and velocity magnitudes do not always accurately match human walking, it is interesting to note that the PDW model shows the same kinematic trends and profile features when compared to human walking under the same conditions.

The PDW, sized with an anthropomorphic model, corresponds reasonably to human walking given that it is passive and does not account for any of the human controls. Although there are variations between human anatomy and PDW model, such as the lack of dorsiflexion and the essential gravity input by a constant decline, that create differences, the normal gait PDW model can be used to approximate general kinematic trajectories of the hip and foot coordination.

PDW Tied-belt and split-belt simulation has its limitations in that the walker becomes unstable at high belt speeds, however at lower speeds it can be used as a good predictor of gait trends with perturbations. To resolve model instability on the split-belt treadmill, an interlimb relative velocity of the same magnitude could be achieved with a simulated splitbelt treadmill running in opposite directions, one pushing the walkers foot forward and one backward. This solution however can lead to unnatural human gait kinematics.

Human and modeled gait perturbations with the GEMS are in very good agreement. In both human and modeled data the heavier GEMS foot compensated for this weight by slowing down during the swing phase. Again, the caricature of the position and velocity graphs are in agreement with the individual's measurements; small decreases and increases in these plots can mainly be described by the PDW model lack of ankle action and the walking on a decline.

Further research can be extended to analyze split-belt treadmill and GEMS in forward and backward directions, asymmetric weighting, or joint damping. Developing this model to predict behavior to force dependent perturbations instead of velocity dependent perturbations is in progress. In addition, clearer metrics for similarity will be developed to further classify the similarities and differences.

\section{ACKNOWLEDGEMENTS}

The authors thank Erin Vasudevan, Rachel German, and Rebecca Glass for their help.

\section{REFERENCES}

[1] D. Reisman, R. Wityk, and A. Bastian, Split-belt treadmill walking adaptation in post-stroke hemiparesis, J. Neurologic Physical Therapy, vol. 29, p. 196, 2005.

[2] T. Lam, M. Anderschitz, and V. Dietz, Contribution of feedback and feedforward strategies to locomotor adaptations, J. Neurophysiol, vol. 95, pp. 766-773, 2006.

[3] S. Morton and A. Bastian, Cerebellar Contributions to Locomotor Adaptations during Splitbelt Treadmill Walking, J. Neurosci., vol. 26, no. 36, pp. 9107-9116, 2006.

[4] T. Lam, C. Wolstenholme, and J. F. Yang, How do infants adapt to loading of the limb during the swing phase of stepping? J. of Neurophysiology, vol. 89, no. 4, pp. 1920-1928, 2003.

[5] D. Reisman, R. Wityk, K. Silver, and A. Bastian, Locomotor adaptation on a split-belt treadmill can improve walking symmetry post-stroke, Brain, vol. 130, no. 7, pp. 1861-1872, 2007.

[6] A. de Groot, R. Decker, and K. B. Reed, Gait Enhancing Mobile Shoe (GEMS) for Rehabilitation, in Joint Eurohaptics Conf. \& Symp. Haptic Interfaces for Virtual Envn. and Teleoperator Sys., 2009, pp. 190-195.

[7] I. Handžić, E. Vasudevan, and K. B. Reed, "Motion Controlled Gait Enhancing Mobile Shoe for Rehabilitation," Proc. of the 12th Intl. Conf. on Rehabilitation Robotics (ICORR), June, 2011.

[8] I. Handžić, E. Vasudevan, and K. B. Reed, "Developing a Gait Enhancing Mobile Shoe to Alter Over-Ground Walking Coordination," Proc. of IEEE Intl. Conf. on Robotics and Automation (ICRA), 2012.

[9] I. Handžić, E. Barno, E. V. Vasudevan, and K. B. Reed. ”Design and Pilot Study of a Gait Enhancing Mobile Shoe," J. of Behavioral Robotics, Vol. 2, Num. 4, pp. 193-201, 2011.

[10] T. McGeer, Passive Dynamic Walking, The International J. of Robotics Research, vol. 9, no. 2, pp. 62-82, 1990.

[11] R. Drillis et al. Body segment parameters: a survey of measurement techniques. National Academy of Sciences, 1964.

[12] C. Honeycutt, J. Sushko, and K. B. Reed, ”Asymmetric Passive Dynamic Walker," Proc. of the 12th Intl. Conf. on Rehabilitation Robotics (ICORR), 2011.

[13] M. Garcia, A. Chatterjee, A. Ruina, and M. Coleman, The simplest walking model: Stability, complexity, and scaling, J. of Biomechanical Engineering, vol. 120, no. 2, p. 281288, 1998.

[14] M.P. Griffin, S. J. Olney, I.D. Mcbride, Role of Symmetry in Gait Performance of Stroke Subjects with Hemiplegia, Gait and Posture, 1996, vol. 3, pp 132-142

[15] J. Perry, Gait Analysis: Normal and pathological function, N. S. Inc., Ed. Thorofare, 1992, vol. 50.

[16] M. Scherer, Gait rehabilitation with body weight-supported treadmill training for a blast injury survivor with traumatic brain injury, Brain Injury, 2007; vol. 21 no.1: pp 93-100

[17] M. Garcia, A. Chatterjee, A. Ruina, and M. Coleman, The simplest walking model: Stability, complexity, and scaling, J. of Biomechanical Engineering, vol. 120, no. 2, p. 281-288, 1998.

[18] A. Goswami, B. Thuilot, and B. Espiau, A Study of the Passive Gait of a Compass-Like Biped Robot, The International J. of Robotics Research, vol. 17, no. 12, pp. 1282-1301, 1998.

[19] V. F. H. Chen,Passive Dynamic Walking with Knees: A Point Foot Model, Master thesis, Massachusetts Institute of Technology, 2005.

[20] R. D. Gregg. Controlled Reduction of a Five-Link 3D Biped with Unactuated Yaw, Proc. of the 5th Intl. Conf. on Descision and Control and European Control, 2011.

[21] R. Gregg, A. Tilton, S. Candido, T. Bretl, and M. Spong. Control and Planning of 3-D Dynamic Walking With Asymptotically Stable Gait Primitive, IEEE Transactions on Robotics, vol. 28, no. 6, 2012.

[22] J. Sushko, C. Honeycutt, K. B. Reed, Prosthesis Design Based on an Asymmetric Passive Dynamic Walker, Proc. of the 4th Intl. Conf. on Biomedical Robotics and Biomechatronics, 2012.

[23] C. Honeycutt. Utilizing a computative model for the design of a passive dynamic walker. Thesis, University of South Florida, 2011.

[24] P. O. Riley, J. Dicharry, J. Franz. U. D. Croce, R. P. Wilder, D. C. Kerrigan. A kinematics and Kinetic of Overground and Treadmill Running, Medicine and Science in Sports and Exercise Applied Sciences, Biodynamics, vol. 10, pp. 1093-1100, 2008.

[25] M. Harris-Love, L. Forrester, R. Macko, K. Silver, G. Smith. Hemiparetic Gait Parameters in Overground Versus Treadmill Walking, Neurorehab and Neural Repair, vol. 15, pp. 105-112, 2001.

[26] A. S. McIntosh, K. T. Beatty, L. N. Dwan, and D. R. Vickers, "Gait dynamics on an inclined walkway." Journal of biomechanics vol. 39., num. 13, 2006, 2491-2502. 